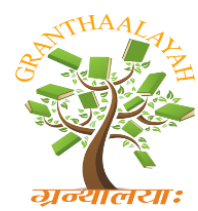

INTERNATIONAL JOURNAL OF RESEARCH GRANTHAALAYAH

A knowledge Repository

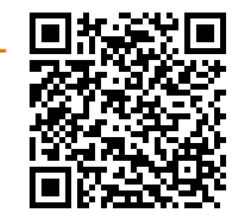

Science

\title{
GENERALIZATIONS OF RAMANUJAN'S RANK FUNCTIONS COLLECTED FROM RAMANUJAN'S LOST NOTEBOOK
}

\author{
Nil Ratan Bhattacharjee ${ }^{* 1}$, Sabuj Das ${ }^{2}$ \\ ${ }^{*}$ Professor, Department of Mathematics, University of Chittagong, BANGLADESH \\ ${ }^{2}$ Senior Lecturer, Department of Mathematics, Raozan University College, BANGLADESH
}

\begin{abstract}
In1916, Srinivasa Ramanujan defined the Mock Theta functions in his lost notebook and unpublished papers. We prove the Mock Theta Conjectures with the help of Dyson's rank and S. Ramanujan's Mock Theta functions. These functions were quoted in Ramanujan's lost notebook and unpublished papers. In1916, Ramanujan stated the theta series in $x$ like $A(x)$, $B(x), C(x), D(x)$. We discuss the Ramanujan's functions with the help of Dyson's rank symbols. These functions are useful to prove the Mock Theta Conjectures. Now first Mock Theta Conjecture is "The number of partitions of $5 n$ with rank congruent to 1 modulo 5 equals the number of partitions of 5n with rank congruent to 0 modulo 5 plus the number of partitions of $n$ with unique smallest part and all other parts the double of the smallest part", and Second Mock Theta Conjecture is "The double of the number of partitions of with rank congruent to 2 modulo 5 equals the sum of the number of partitions of with rank congruent to 0 and congruent to1 modulo 5, and the sum of one and the number of partitions of $n$ with unique smallest part and all other parts $\square$ one plus the double of the smallest part". This paper shows how to prove the Theorem 1.3 with the help of Dyson's rank symbols $N(0,5,5 n+1), N(2,5,5 n+1)$ and shows how to prove the Theorem 1.4 with the help of Ramanujan's theta series and Dyson's rank symbols $N(1,5,5 n+2), N(2,5,5 n+2)$ respectively.
\end{abstract}

Keywords:

Congruent to; Dyson's rank; Mock Theta, Modulo; Ramanujan's lost notebook.

Cite This Article: Nil Ratan Bhattacharjee, and Sabuj Das, "GENERALIZATIONS OF RAMANUJAN'S RANK FUNCTIONS COLLECTED FROM RAMANUJAN'S LOST NOTEBOOK" International Journal of Research - Granthaalayah, Vol. 4, No. 3 (2016): 1-20.

\section{INTRODUCTION}

In this paper we give the definitions of $\pi$, rank of partition, $N(m, n), N(m, t, n), \mathrm{z},(x)_{\infty},(z x)_{\infty},\left(x^{n}\right)_{m}$, $\left(x^{k} ; x^{5}\right)_{m}, \rho_{0}(n), \rho_{1}(n), \beta_{1}(n)$ and $\beta_{2}(n)$ which are collected from Ramanujan's Lost Notebook VI and generate the generating functions of Dyson's Rank. We generate the generating functions for $\mathrm{A}(\mathrm{x}), \mathrm{D}(\mathrm{x}), \phi(x), \Psi(x), \rho_{0}(n)$ and $\rho_{1}(n)$ and prove the two Theorems first and second mock theta 
conjectures respectively. We give two numerical examples which are related to first and second mock theta conjectures respectively when $n=1$. We generate the generating functions for $\beta_{1}(n)$ and $\beta_{2}(n)$ collected from Ramanujan's Lost notebook V1. We prove the Theorem 3 in terms of $\beta_{1}(n)$ with the help of Ramanujan's identities and prove the Theorem 4 in terms of $\beta_{2}(n)$ with the help of Dyson's rank symbols and Ramanujan's identities.

\section{SOME RELATED DEFINITIONS}

$\pi$ : A partition.

Rank of partition: The largest part of a partition $\pi$ minus the number of parts of $\pi$. $N(m, n)$ : The number of partitions of $n$ with rank $m$.

$N(m, t, n)$ : The number of partition of $n$ with rank congruent to $m$ modulo $t$.

$\mathrm{z}$ : The set of complex numbers.

$(x)_{\infty}$ : The product of infinite factors is defined as follows:

$(x)_{\infty}=(1-x)\left(1-x^{2}\right)\left(1-x^{3}\right) \ldots \infty$ where $|x|<1$.

$(z x)_{\infty}$ : The product of infinite factors is defined as follows:

$(z x)_{\infty}=(1-z x)\left(1-z x^{2}\right)\left(1-z x^{3}\right) \ldots \infty$.

$\left(x^{n}\right)_{m}$ : The product of $m$ factors is defined as follows:

$\left(x^{n}\right)_{m}=\left(1-x^{n}\right)\left(1-x^{n+1}\right)\left(1-x^{n+2}\right) \ldots\left(1-x^{n+m-1}\right)$.

$\left(x^{k} ; x^{5}\right)_{m}$ : The product of $m$ factors is defined as follows:

$\left(x^{k} ; x^{5}\right)_{m}=\left(1-x^{k}\right)\left(1-x^{k+5}\right)\left(1-x^{k+10}\right) \ldots\left(1-x^{k+(m-1) 5}\right)$.

$\rho_{0}(n)$ : The number of partitions of $n$ with unique smallest part and all other parts $\leq$ the double of the smallest part.

$\rho_{1}(n)$ : The number of partitions of $n$ with unique smallest part and all other parts $\leq$ one plus the double of the smallest part.

$\beta_{1}(n)$ : The number of partitions of $n$ into 1 's and parts congruent to 0 or -1 modulo 5 with the largest part $\equiv 0(\bmod 5) \leq 5$ times the number of 1 's.

$\beta_{2}(n)$ : The number of partitions of $n$ into 2's and parts congruent to 0 or -2 modulo 5 with the largest part $\equiv 0(\bmod 5) \leq 5$ times the number of 2 's.

\section{RANK OF A PARTITION [DYSON (1944)]}

The rank of a partition is defined as the largest part of a partition $\pi$ minus the number of parts of the partition $\pi$.Thus the partition $5+4+1+1$ of 11 has rank $5-4=1$. The rank of a partition of 11 belongs to any one of the residues $(\bmod 11)$ and we have exactly 11 residues.

\subsection{The generating function for $N(m, n)$ [Garvan (2013)] is given below;}

We get $N(m, n)$ is the number of partitions of $n$ with rank $m$, like

The ranks of partitions of 3 are given in the Table- 1

\begin{tabular}{|l|l|l|}
\hline$n$ & Type of partitions & Rank \\
\hline 3 & 3 & $3-1=2$ \\
\hline
\end{tabular}




\begin{tabular}{|l|l|}
\hline $2+1$ & $2-2=0$ \\
\hline $1+1+1$ & $1-3=-2$ \\
\hline
\end{tabular}

The ranks of partitions of 4 are given in the Table- 2

\begin{tabular}{|l|l|l|l|}
\hline$n$ & Type of partitions & Rank & Rank (mod 5) \\
\hline 4 & 4 & $4-1=3$ & $\equiv 3$ \\
\cline { 2 - 4 } & $3+1$ & $3-2=1$ & $\equiv 1$ \\
\cline { 2 - 4 } & $2+2$ & $2-2=0$ & $\equiv 0$ \\
\cline { 2 - 4 } & $2+1+1$ & $2-3=-1$ & $\equiv 4$ \\
\cline { 2 - 4 } & $1+1+1+1$ & $1-4=-3$ & $\equiv 2$ \\
\hline
\end{tabular}

We can write an expression as;

$$
1+x+\left(z+z^{-1}\right) x^{2}+\left(1+z^{2}+z^{-2}\right) x^{3}+\left(1+z+z^{-1}+z^{3}+z^{-3}\right) x^{4}+\ldots
$$

[The exponent of $z$ being the rank of partitions of any positive integer of $n$ ]

$$
=\sum_{n=0}^{\infty} \sum_{m=-\infty}^{\infty} N(m, n) z^{m} x^{n}
$$

or, $1+\frac{x}{(1-z x)\left(1-z^{-1} x\right)}+\frac{x^{4}}{(1-z x)\left(1-z x^{2}\right)\left(1-z^{-1} x\right)\left(1-z^{-1} x^{2}\right)}+. .=\sum_{n=0}^{\infty} \sum_{m=-\infty}^{\infty} N(m, n) z^{m} x^{n}$

or, $\sum_{n=0}^{\infty} \frac{x^{n^{2}}}{(z x)_{n}\left(z^{-1} x\right)_{n}}=\sum_{n=0}^{\infty} \sum_{m=-\infty}^{\infty} N(m, n) z^{m} x^{n}$, where the power of $\mathrm{z}$ are the rank of $\mathrm{n}$.

$\therefore \sum_{n=0}^{\infty} \sum_{m=-\infty}^{\infty} N(m, n) z^{m} x^{n}=\sum_{n=0}^{\infty} \frac{x^{n^{2}}}{(z x)_{n}\left(z^{-1} x\right)_{n}}$.

We can generate the generating function for $N(m, n)$ in another way

\subsection{The generating function for $N(1, n)$ is given below;}

We get, $N(1, n)$ is the number of partitions of $n$ with rank 1 given in the Table-3

\begin{tabular}{|l|l|l|}
\hline$n$ & Type of partitions & $N(1, n)$ \\
\hline 1 & none & 0 \\
\hline 2 & 2 & 1 \\
\hline 3 & none & 0 \\
\hline 4 & $3+1$ & 1 \\
\hline 5 & $3+2$ & 1 \\
\hline 6 & $4+1+1,3+3$ & 2 \\
$\ldots$ & $\ldots$ &
\end{tabular}

We can write an expression as;

$N(1,0)+N(1,1) x+N(1,2) x^{2}+N(1,3) x^{3}+N(1,4) x^{4}+\ldots$, where $N(1,0)=0$

$=0+x^{2}+0+x^{4}+x^{5}+2 x^{6}+\ldots$

$=\left(x^{2}-x^{3}-x^{7}+x^{9}+\ldots\right)\left(1+x+2 x^{2}+3 x^{3}+5 x^{4}+\ldots\right)$

$=\sum_{n=1}^{\infty}(-1)^{n-1} x^{\frac{n}{2}(3 n-1)+n}\left(1-x^{n}\right) \prod_{j=1}^{\infty} \frac{1}{1-x^{j}} \quad[\operatorname{Garvan}(1988)]$ 
$\therefore \sum_{n=1}^{\infty}(-1)^{n-1} x^{\frac{n}{2}(3 n-1)+n}\left(1-x^{n}\right) \prod_{j=1}^{\infty} \frac{1}{1-x^{j}}=\sum_{n=0}^{\infty} N(1, n) x^{n}$

3.3.The generating function for $N(-1, n)$ is given below;

We get, $N(-1, n)$ is the number of partitions of $n$ with rank -1 given in the Table- 4

\begin{tabular}{|l|l|l|}
\hline$n$ & Type of partitions & $N(-1, n)$ \\
\hline 1 & none & 0 \\
\hline 2 & $1+1$ & 1 \\
\hline 3 & none & 0 \\
\hline 4 & $2+1+1$ & 1 \\
\hline 5 & $2+2+1$ & 1 \\
\hline 6 & $3+1+1+1,2+2+2$ & 2 \\
$\ldots$ & $\ldots$ & $\ldots$ \\
\hline
\end{tabular}

We can write an expression as;

$N(-1,0)+N(-1,1) x+N(-1,2) x^{2}+N(-1,3) x^{3}+N(-1,4) x^{4}+\ldots$, where $N(-1,0)=0$

$=0+x^{2}+0+x^{4}+x^{5}+2 x^{6}+\ldots$

$=\left(x^{2}-x^{3}-x^{7}+x^{9}+\ldots\right)\left(1+x+2 x^{2}+3 x^{3}+5 x^{4}+\ldots\right)$

$=\sum_{n=1}^{\infty}(-1)^{n-1} x^{\frac{n}{2}(3 n-1)+n}\left(1-x^{n}\right) \prod_{j=1}^{\infty} \frac{1}{1-x^{j}} \quad$ [Garvan (2013)]

$\therefore \sum_{n=1}^{\infty}(-1)^{n-1} x^{\frac{n}{2}(3 n-1)+n}\left(1-x^{n}\right) \prod_{j=1}^{\infty} \frac{1}{1-x^{j}}=\sum_{n=0}^{\infty} N(-1, n) x^{n}$.

From (3.2a) and (3.3a) we get, $N(1, n)=N(-1, n)$, [by equating the coefficient of $x^{n}$ ].

\subsection{The generating function for $N(2, n)$ is given below;}

We get, $N(2, n)$ is the number of partitions of $n$ with rank 2 given in the Table- 5

We can write an expression as;

\begin{tabular}{|l|l|l|}
\hline$n$ & Type of partitions & $N(2, n)$ \\
\hline 1 & none & 0 \\
\hline 2 & none & 0 \\
\hline 3 & 3 & 1 \\
\hline 4 & none & 0 \\
\hline 5 & $4+1$ & 1 \\
\hline$\ldots$ & $\ldots$ & $\ldots$ \\
\hline
\end{tabular}

$N(2,0)+N(2,1) x+N(2,2) x^{2}+N(2,3) x^{3}+N(2,4) x^{4}+\ldots$, where $N(2,0)=0$

$=0+x^{3}+x^{5}+x^{6}+\ldots$

$=\left(x^{3}-x^{4}-x^{9}+\ldots\right)\left(1+x+2 x^{2}+3 x^{3}+5 x^{4}+\ldots\right)$

$=\sum_{n=1}^{\infty}(-1)^{n-1} x^{\frac{n}{2}(3 n-1)+2 n}\left(1-x^{n}\right) \prod_{j=1}^{\infty} \frac{1}{1-x^{j}}=\sum_{n=0}^{\infty} N(2, n) x^{n}$. 
$\therefore \sum_{n=1}^{\infty}(-1)^{n-1} x^{\frac{n}{2}(3 n-1)+2 n}\left(1-x^{n}\right) \prod_{j=1}^{\infty} \frac{1}{1-x^{j}}=\sum_{n=0}^{\infty} N(2, n) x^{n} \ldots$

From (3.2a),(3.3a) and (3.4a) we can conclude that,

$$
\sum_{n=1}^{\infty}(-1)^{n-1} x^{\frac{n}{2}(3 n-1)+|m| n}\left(1-x^{n}\right) \prod_{j=1}^{\infty} \frac{1}{1-x^{j}}=\sum_{n=0}^{\infty} N(m, n) x^{n} .
$$

Now we can generate the generating function for $N(m, t, n)$ [Atkin et al. (1954)]:

We get $N(m, t, n)$ is the number of partitions of $n$ with rank congruent to $m$ modulo $t$.

\subsection{The generating function for $N(0,5, n)$ is given below:}

We get, $N(0,5, n)$ is the number of partitions of $n$ with rank congruent to 0 modulo 5 given in the Table- 6

\begin{tabular}{|l|l|l|}
\hline$n$ & Type of partitions & $N(0,5, n)$ \\
\hline 1 & 1 & 1 \\
\hline 2 & none & 0 \\
\hline 3 & $2+1$ & 1 \\
\hline 4 & $2+2$ & 1 \\
\hline$\ldots$ & $\ldots$ & $\ldots$ \\
\hline
\end{tabular}

We can write an expression as;

$N(0,5,0)+N(0,5,1) x+N(0,5,2) x^{2}+N(0,5,3) x^{3}+\ldots$, where $N(0,5,0)=0$

$=0+x+x^{3}+x^{4}+\ldots$

$=\sum_{n=1}^{\infty}(-1)^{n} x^{\frac{n}{2}(3 n+1)}\left(1+x^{5 n}\right)\left(1-x^{5 n}\right)^{-1}\left(1+x+2 x^{2}+3 x^{3}+5 x^{4}+\ldots\right)$

$\therefore \sum_{n=1}^{\infty}(-1)^{n} x^{\frac{n}{2}(3 n+1)}\left(1+x^{5 n}\right)\left(1-x^{5 n}\right)^{-1} \prod_{j=1}^{\infty}\left(1-x^{j}\right)^{-1}=\sum_{n=0}^{\infty} N(0,5, n) x^{n} \ldots$

\subsection{The generating function for $N(3,5, n)$ is given below:}

We get, $N(3,5, n)$ is the number of partitions of $n$ with rank congruent to 3 modulo 5 given in the Table-7

\begin{tabular}{|l|l|l|}
\hline$n$ & Type of partitions & $N(3,5, n)$ \\
\hline 1 & none & 0 \\
\hline 2 & none & 0 \\
\hline 3 & $1+1+1$ & 1 \\
\hline 4 & 4 & 1 \\
\hline$\ldots$ & $\ldots$ & $\ldots$ \\
\hline
\end{tabular}

We can write an expression as;

$N(3,5,0)+N(3,5,1) x+N(3,5,2) x^{2}+N(3,5,3) x^{3}+\ldots$, where $N(3,5,0)=0$

$=0+0+x^{3}+x^{4}+\ldots$

$=\sum_{n=1}^{\infty}(-1)^{n} x^{\frac{n}{2}(3 n+1)}\left(x^{3 n}+x^{2 n}\right)\left(1-x^{5 n}\right)^{-1}\left(1+x+2 x^{2}+3 x^{3}+\ldots\right)$ 
$\therefore \sum_{n=1}^{\infty}(-1)^{n} x^{\frac{n}{2}(3 n+1)}\left(x^{3 n}+x^{2 n}\right)\left(1-x^{5 n}\right)^{-1} \prod_{j=1}^{\infty} \frac{1}{1-x^{j}}=\sum_{n=0}^{\infty} N(3,5, n) x^{n} \ldots$

\subsection{The generating function for $N(4,7, n)$ is given below:}

We get, $N(4,7, n)$ is the number of partitions of $n$ with rank congruent to 4 modulo 7 given in the Table- 8

\begin{tabular}{|l|l|l|}
\hline$n$ & Type of partitions & $N(4,7, n)$ \\
\hline 1 & none & 0 \\
\hline 2 & none & 0 \\
\hline 3 & none & 0 \\
\hline 4 & $1+1+1+1$ & 1 \\
\hline 5 & 5 & 1 \\
\hline$\ldots$ & $\ldots$ & $\ldots$ \\
\hline
\end{tabular}

$$
\begin{aligned}
& \text { We can } \quad \text { write } \\
& N(4,7,0)+N(4,7,1) x+N(4,7,2) x^{2}+N(4,7,3) x^{3}+N(4,7,4) x^{4}+\ldots+N(4,7,8) x^{8}+\ldots, \\
& \quad \text { where } N(4,7,0)=0 \\
& =0+0+x^{4}+x^{5}+x^{6}+\ldots \\
& =\sum_{n=1}^{\infty}(-1)^{n} x^{\frac{n}{2}(3 n+1)}\left(x^{4 n}+x^{3 n}\right)\left(1-x^{7 n}\right)^{-1}\left[1+x+2 x^{2}+3 x^{3}+5 x^{4}+\ldots\right] \\
& \therefore \sum_{n=1}^{\infty}(-1)^{n} x^{\frac{n}{2}(3 n+1)}\left(x^{4 n}+x^{3 n}\right)\left(1-x^{7 n}\right)^{-1} \prod_{j=1}^{\infty} \frac{1}{1-x^{j}}=\sum_{n=0}^{\infty} N(4,7, n) x^{n} \ldots \quad \text { (3.7a). }
\end{aligned}
$$

From (3.5a),(3.6a) and (3.7a) we can conclude that,

$$
\therefore \sum_{n=1}^{\infty}(-1)^{n} x^{\frac{n}{2}(3 n+1)}\left(x^{m n}+x^{n(t-m)}\right)\left(1-x^{t n}\right)^{-1} \prod_{j=1}^{\infty} \frac{1}{1-x^{j}}=\sum_{n=0}^{\infty} N(m, t, n) x^{n} \text {, }
$$

which shows that all the coefficients of $x^{-n}$ (where $n$ is any positive integer) are zero.

Now we define the generating functions;

$$
r_{a}(d) \text { for } N(a, t, t n+d) \text {, where } r_{a}(d)=r_{a}(d, t)=\sum_{n=0}^{\infty} N(a, t, t n+d) x^{n}, \text { and }
$$$$
r_{a, b}(d)=r_{a, b}(d, t)=r_{a}(d)-r_{b}(d)=\sum_{n=0}^{\infty}\{N(a, t, t n+d)-N(b, t, t n+d)\} x^{n}
$$

Result 1: $N(k, 11,11 n+6)=\frac{P(11 n+6)}{11} ; 0 \leq k \leq 10$.

Proof: We get the list of all partitions of 17 is;

$17,16+1,15+2,15+1+1,14+3,14+2+1,14+1+1+1,13+4,13+3+1,13+2+2,13+2+1+1,13+1+1+1+1,1$ $2+5,12+4+1, \ldots, 1+1+1+1+1+1+1+1+1+1+1+1+1+1+1+1+1$.

Hence, there are 297 partitions i.e., $\mathrm{P}(17)=297$.

Now their corresponding ranks are;

$16,14,13,12,12,11,10,11,10, \ldots .,-10,-11,-12,-12,-13,-14,-16$. 
$N(0,11,17)=N(1,11,17)=N(2,11,17)=N(3,11,17)=N(4,11,17)=\ldots$

$=N(9,11,17)=N(10,11,17)=27=\frac{P(17)}{11}$.

So, we can say that; $N(k, 11,17)=\frac{P(17)}{11} ; 0 \leq k \leq 10$.

Generally we can conclude that; $N(k, 11,11 n+6)=\frac{P(11 n+6)}{11} ; 0 \leq k \leq 10$. Hence the Result.

Similarly we can see the Result 2: $N(k, 5,5 n+4)=\frac{P(5 n+4)}{5} ; 0 \leq k \leq 4$.

And the Result 3: $N(k, 7,7 n+5)=\frac{P(7 n+5)}{7} ; 0 \leq k \leq 6$.

Remark 1 [Atkin et al. (1954)] : $N(m, t, n)=N(t-m, t, n)$

Proof: The generating function for $N(1,5, n)$ is given below:

We get, $N(1,5, n)$ is the number of partitions of $n$ with rank congruent to 1 modulo 5 given in the Table- 9

\begin{tabular}{|l|l|l|}
\hline$n$ & Type of partitions & $N(1,5, n)$ \\
\hline 1 & none & 0 \\
\hline 2 & 2 & 1 \\
\hline 3 & none & 0 \\
\hline 4 & $3+1$ & 1 \\
\hline$\ldots$ & $\ldots$ & $\ldots$ \\
\hline
\end{tabular}

We can write an expression as;

$N(1,5,0)+N(1,5,1) x+N(1,5,2) x^{2}+N(1,5,3) x^{3}+\ldots$, where $N(1,5,0)=0$

$=0+0+x^{2}+0+x^{4}+\ldots$

$=\sum_{n=1}^{\infty}(-1)^{n} x^{\frac{n}{2}(3 n+1)}\left(x^{n}+x^{4 n}\right)\left(1-x^{5 n}\right)^{-1} \prod_{j=1}^{\infty} \frac{1}{1-x^{j}}$

$\therefore \sum_{n=1}^{\infty}(-1)^{n} x^{\frac{n}{2}(3 n+1)}\left(x^{n}+x^{4 n}\right)\left(1-x^{5 n}\right)^{-1} \prod_{j=1}^{\infty}\left(1-x^{j}\right)^{-1}=\sum_{n=0}^{\infty} N(1,5, n) x^{n}$.

Again the generating function for $N(4,5, n)$ is given below:

We get, $N(4,5, n)$ is the number of partitions of $n$ with rank congruent to 1 modulo 5 given in the Table- 10

\begin{tabular}{|l|l|l|}
\hline$n$ & Type of partitions & $N(4,5, n)$ \\
\hline 1 & none & 0 \\
\hline 2 & 2 & 1 \\
\hline 3 & none & 0 \\
\hline 4 & $3+1$ & 1 \\
\hline$\ldots$ & $\ldots$ & $\ldots$ \\
\hline
\end{tabular}

We can write an expression as;

$N(4,5,0)+N(4,5,1) x+N(4,5,2) x^{2}+N(4,5,3) x^{3}+\ldots$, where $N(4,5,0)=0$

$=0+0+x^{2}+0+x^{4}+\ldots$ 
$=\sum_{n=1}^{\infty}(-1)^{n} x^{\frac{n}{2}(3 n+1)}\left(x^{n}+x^{4 n}\right)\left(1-x^{5 n}\right)^{-1} \prod_{j=1}^{\infty} \frac{1}{1-x^{j}}$
$\therefore \sum_{n=1}^{\infty}(-1)^{n} x^{\frac{n}{2}(3 n+1)}\left(x^{n}+x^{4 n}\right)\left(1-x^{5 n}\right)^{-1} \prod_{j=1}^{\infty}\left(1-x^{j}\right)^{-1}=\sum_{n=0}^{\infty} N(4,5, n) x^{n}$.

From (3.7b) and (3.7c) we get;

$N(1,5, n)=N(4,5, n)$

$\therefore N(1,5, n)=N(5-1,5, n)$.

It follows that, $N(m, 5, n)=N(5-m, 5, n) ; m=[0,4]$.

Generally, we can conclude that, $N(m, t, n)=N(t-m, t, n)$. Hence the Remark.

Remark 2 [Garvan (1988)]: $N(1,5,5 n+1)=N(2,5,5 n+1)$

Proof: The generating function for $N(2,5, n)$ is given below:

We get, $N(2,5, n)$ is the number of partitions of $n$ with rank congruent to 2 modulo 5 given in the Table-11

\begin{tabular}{|l|l|l|}
\hline$n$ & Type of partitions & $N(2,5, n)$ \\
\hline 1 & none & 0 \\
\hline 2 & none & 0 \\
\hline 3 & 3 & 1 \\
\hline 4 & $1+1+1+1$ & 1 \\
\hline$\ldots$ & $\ldots$ & $\ldots$ \\
\hline
\end{tabular}

We can write an expression as;

$N(2,5,0)+N(2,5,1) x+N(2,5,2) x^{2}+N(2,5,3) x^{3}+\ldots$, where $N(2,5,0)=0$

$=0+0+x^{3}+x^{4}+\ldots$

$=\sum_{n=1}^{\infty}(-1)^{n} x^{\frac{n}{2}(3 n+1)}\left(x^{2 n}+x^{3 n}\right)\left(1-x^{5 n}\right)^{-1} \prod_{j=1}^{\infty} \frac{1}{1-x^{j}}$

$\therefore \sum_{n=1}^{\infty}(-1)^{n} x^{\frac{n}{2}(3 n+1)}\left(x^{2 n}+x^{3 n}\right)\left(1-x^{5 n}\right)^{-1} \prod_{j=1}^{\infty} \frac{1}{1-x^{j}}=\sum_{n=0}^{\infty} N(2,5, n) x^{n}$

$=x^{3}+x^{4}+2 x^{6}+2 x^{7}+5 x^{8}+6 x^{9}+8 x^{10}+11 x^{11}+\ldots$

$=x^{3}+x^{4}+N(2,5,6) x^{6}+2 x^{7}+5 x^{8}+6 x^{9}+8 x^{10}+N(2,5,11) x^{11}+\ldots$

Again from (3.7b) we get;

$\sum_{n=1}^{\infty}(-1)^{n} x^{\frac{n}{2}(3 n+1)}\left(x^{n}+x^{4 n}\right)\left(1-x^{5 n}\right)^{-1} \prod_{j=1}^{\infty}\left(1-x^{j}\right)^{-1}=\sum_{n=0}^{\infty} N(1,5, n) x^{n}$.

$=x^{2}+0 . x^{3}+x^{4}+2 x^{5}+2 x^{6}+\ldots+9 x^{10}+11 x^{11}+\ldots$

$=x^{2}+x^{4}+2 x^{5}+N(1,5,6) x^{6}+\ldots+9 x^{10}+N(1,5,11) x^{11}+\ldots$

From (3.7d) and (3.7e) we get;

$N(1,5,6)=N(2,5,6), N(1,5,11)=N(2,5,11), N(1,5,16)=N(2,5,16), \ldots$

Generally, we can conclude that; $N(1,5,5 n+1)=N(2,5,5 n+1)$. Hence the Remark.

Similarly we can see the $\operatorname{Remark} 3: N(0,5,5 n+2)=N(2,5,5 n+2)$. 
Remark 4: $N(0,7,7 n+4)=N(1,7,7 n+4)$

Proof: The generating function for $N(0,7, n)$ is given below:

We get, $N(0,7, n)$ is the number of partitions of $n$ with rank congruent to 0 modulo 7 given in the Table-12

\begin{tabular}{|l|l|l|}
\hline$n$ & Type of partitions & $N(0,7, n)$ \\
\hline 1 & 1 & 1 \\
\hline 2 & none & 0 \\
\hline 3 & $2+1$ & 1 \\
\hline 4 & $2+2$ & 1 \\
\hline$\ldots$ & $\ldots$ & $\ldots$ \\
\hline
\end{tabular}

We can write an expression as;

$$
\begin{aligned}
& N(0,7,0)+N(0,7,1) x+N(0,7,2) x^{2}+N(0,7,3) x^{3}+N(0,7,4) x^{4}+\ldots+N(0,7,11) x^{11}+\ldots \\
& \quad \text { where } N(0,7,0)=0 \\
& =x+x^{3}+x^{4}+x^{5}+x^{6}+3 x^{7}+\ldots+8 x^{11}+\ldots \\
& =\sum_{n=1}^{\infty}(-1)^{n} x^{\frac{n}{2}(3 n+1)}\left(1+x^{7 n}\right)\left(1-x^{7 n}\right)^{-1} \prod_{j=1}^{\infty} \frac{1}{1-x^{j}} \\
& \therefore \sum_{n=1}^{\infty}(-1)^{n} x^{\frac{n}{2}(3 n+1)}\left(1+x^{7 n}\right)\left(1-x^{7 n}\right)^{-1} \prod_{j=1}^{\infty} \frac{1}{1-x^{j}}=\sum_{n=0}^{\infty} N(0,7, n) x^{n} \ldots
\end{aligned}
$$

Again, the generating function for $N(1,7, n)$ is given below:

We get, $N(1,7, n)$ is the number of partitions of $n$ with rank congruent to 1 modulo 7 given in the Table-13

\begin{tabular}{|l|l|l|}
\hline$n$ & Type of partitions & $N(1,7, n)$ \\
\hline 1 & none & 0 \\
\hline 2 & 2 & 1 \\
\hline 3 & none & 0 \\
\hline 4 & $3+1$ & 1 \\
\hline$\ldots$ & $\ldots$ & $\ldots$ \\
\hline
\end{tabular}

We make the expression;

$$
\begin{aligned}
& N(1,7,0)+N(1,7,1) x+N(1,7,2) x^{2}+N(1,7,3) x^{3}+N(1,7,4) x^{4}+\ldots+N(1,7,11) x^{11}+\ldots, \\
& \quad \text { where } N(1,7,0)=0 \\
& =0+x^{2}+x^{4}+x^{5}+\ldots+8 x^{11}+\ldots \\
& =\sum_{n=1}^{\infty}(-1)^{n} x^{\frac{n}{2}(3 n+1)}\left(x^{n}+x^{6 n}\right)\left(1-x^{7 n}\right)^{-1}\left[1+x+2 x^{2}+3 x^{3}+5 x^{4}+\ldots\right] \\
& \therefore \sum_{n=1}^{\infty}(-1)^{n} x^{\frac{n}{2}(3 n+1)}\left(x^{n}+x^{6 n}\right)\left(1-x^{7 n}\right)^{-1} \prod_{j=1}^{\infty} \frac{1}{1-x^{j}}=\sum_{n=0}^{\infty} N(1,7, n) x^{n} .
\end{aligned}
$$

From (3.7f) and (3.7g) we get;

$N(0,7,4)=N(1,7,4), N(0,7,11)=N(1,7,11)$

Generally, we can conclude that; $N(0,7,7 n+4)=N(1,7,7 n+4)$. Hence the Remark . 
Similarly we can see the Remark 5: $N(2,7,7 n)=N(3,7,7 n)$.

Remark 6: $N(2,7,7 n+1)=N(3,7,7 n+1)$.

Remark 7: $N(0,7,7 n+2)=N(3,7,7 n+2)$.

And the Remark 8: $N(1,7,7 n+3)=N(3,7,7 n+3)$.

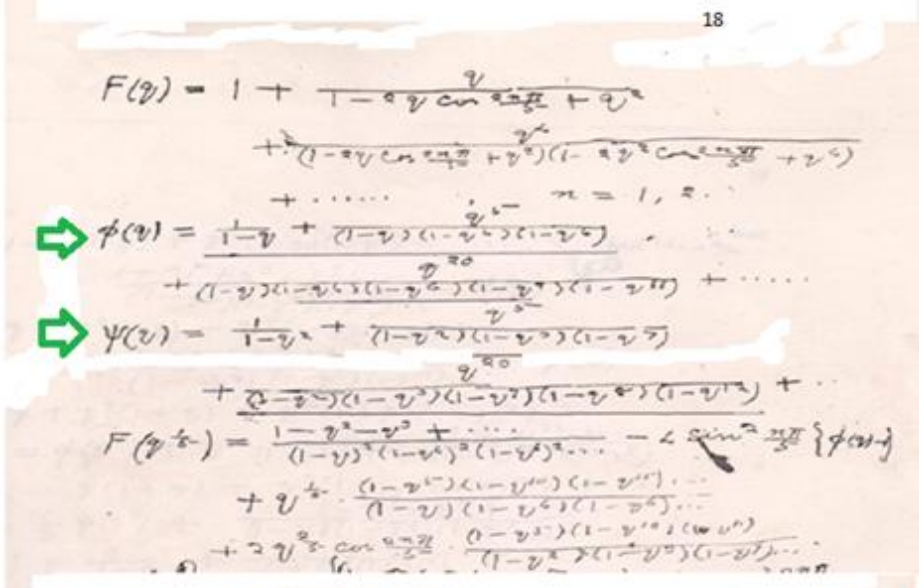

Fig. 1. The rank functions $\phi(\vartheta)$ and $\psi(\nu)$ are stated by Ramanujan in his Lost Notebook. (1916).

Corollary 1: Prove that $\phi(x)=r_{1,2}(0)$.

Proof: We get,

$$
\begin{aligned}
\phi(x) & =-1+\left\{\frac{1}{1-x}+\frac{x^{5}}{(1-x)\left(1-x^{4}\right)\left(1-x^{6}\right)}+\frac{x^{20}}{(1-x)\left(1-x^{4}\right)\left(1-x^{6}\right)\left(1-x^{9}\right)\left(1-x^{11}\right)}+\ldots \infty\right\} \\
& =-1+\left(1+x+x^{2}+\ldots \infty\right)+x^{5}\left(1+x+x^{2}+\ldots \infty\right)\left(1+x^{4}+\ldots \infty\right)\left(1+x^{6}+\ldots \infty\right)+\ldots \infty \\
& =x+x^{2}+x^{3}+x^{4}+2 x^{5}+2 x^{6}+2 x^{7}+2 x^{8}+\ldots \infty \\
& =(o-o)+(2-1) x+(9-8) x^{2}+\ldots \infty \\
& =\{N(1,5,0)-N(2,5,0)\}+\{N(1,5,5)-N(2,5,5)\} x+\{N(1,5,10)-N(2,5,10)\} x^{2}+\ldots . \\
& =\sum_{n=0}^{\infty}\{N(1,5,5 n)-N(2,5,5 n)\} x^{n} \\
& =r_{1,2}(0) . \\
\therefore & \phi(x)=r_{1,2}(0) . \text { Hence the Corollary. }
\end{aligned}
$$




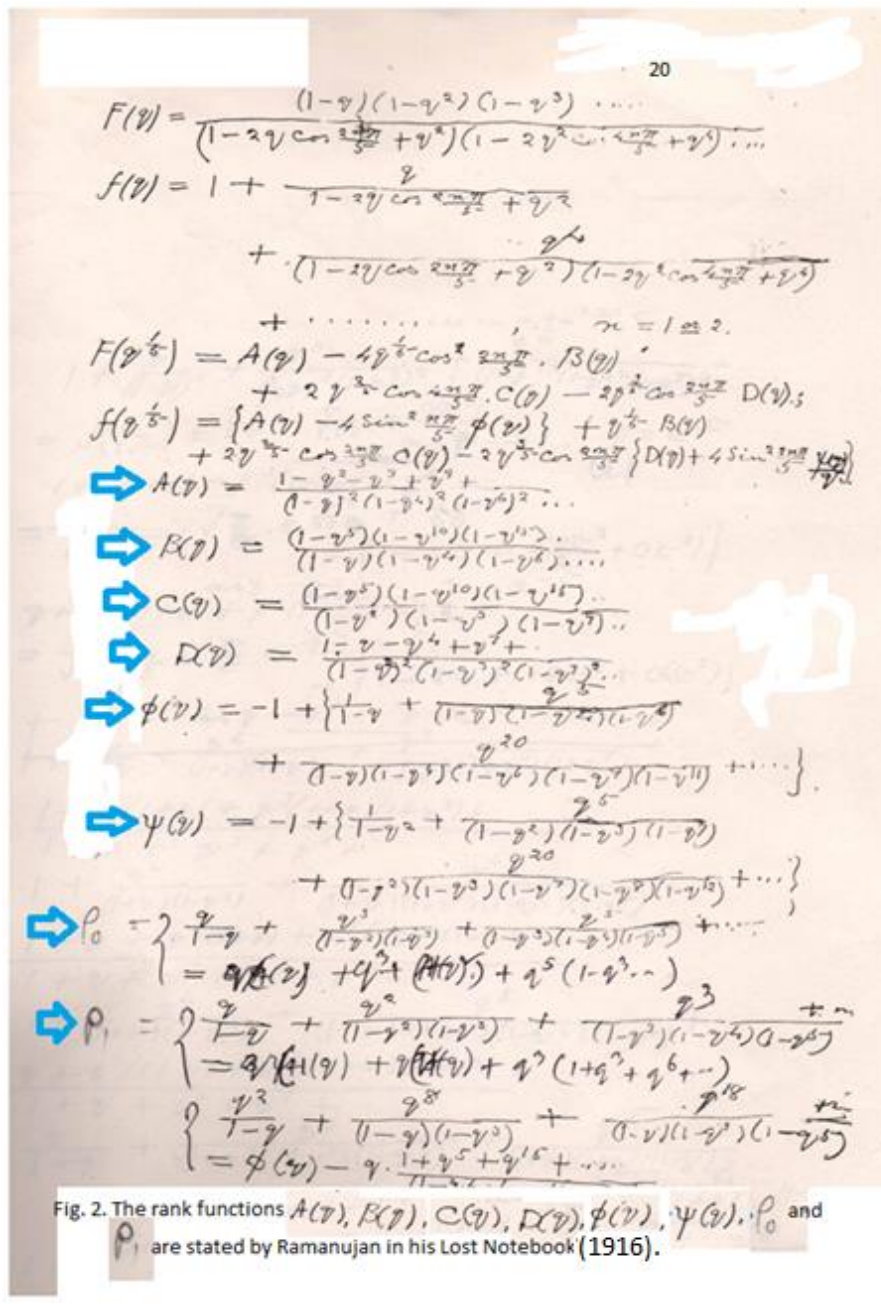

Corollary 2 [Andrews et al. (1989)]: Prove that $\mathrm{A}(x)=1+r_{0,2}(0)+2 r_{1,2}(0)$

Proof: We get, $A(x)=\prod_{n=1}^{\infty} \frac{\left(1-x^{5 n-3}\right)\left(1-x^{5 n-2}\right)\left(1-x^{5 n}\right)}{\left(1-x^{5 n-4}\right)^{2}\left(1-x^{5 n-1}\right)^{2}}$, [Ramanujan's lost notebook(1916)]

$$
\begin{aligned}
&=\frac{1-x^{2}-x^{3}+x^{9}+\ldots \infty}{(1-x)^{2}\left(1-x^{4}\right)^{2}\left(1-x^{6}\right)^{2} \ldots \infty} \\
&=\left(1-x^{2}-x^{3}+x^{9}+\ldots \infty\right)\left(1+2 x+3 x^{2}+\ldots \infty\right)\left(1+2 x^{4}+3 x^{8}+\ldots \infty\right) \ldots \infty \\
&= 1+2 x+2 x^{2}+x^{3}+2 x^{4}+\ldots \infty \\
&= 1+\sum_{n=0}^{\infty}\{N(0,5,5 n)-N(2,5,5 n)+2 N(1,5,5 n)-2 N(2,5,5 n)\} x^{n} \\
&= 1+\sum_{n=0}^{\infty}\{N(0,5,5 n)-N(2,5,5 n)\} x^{n}+2 \sum_{n=0}^{\infty}\{N(1,5,5 n)-N(2,5,5 n)\} x^{n} \\
&= 1+r_{0,2}(0)+2 r_{1,2}(0) . \\
& \therefore \mathrm{A}(x)=1+r_{0,2}(0)+2 r_{1,2}(0) . \text { Hence the Corollary. }
\end{aligned}
$$

Corollary 3[Andrews (1979)]: Prove that $D(x)=r_{0,1}(3)+r_{0,2}(3)$ 
Proof: We get, $D(x)=\prod_{n=1}^{\infty} \frac{\left(1-x^{5 n-4}\right)\left(1-x^{5 n-1}\right)\left(1-x^{5 n}\right)}{\left(1-x^{5 n-3}\right)^{2}\left(1-x^{5 n-2}\right)^{2}}$, [Ramanujan's lost notebook(1916)]

$$
\begin{aligned}
& =\frac{1-x-x^{4}+x^{7}+\ldots \infty}{\left(1-x^{2}\right)^{2}\left(1-x^{3}\right)^{2}\left(1-x^{7}\right)^{2} \ldots \infty} \\
& =\left(1-x-x^{4}+x^{7}+\ldots \infty\right)\left(1+2 x^{2}+3 x^{4}+\ldots \infty\right)\left(1+2 x^{3}+\ldots \infty\right)\left(1+2 x^{7}+\ldots \infty\right) \ldots \infty \\
& =1-x+2 x^{2}+0 \cdot x^{3}+\ldots \infty \\
& =\sum_{n=0}^{\infty}\{N(0,5,5 n+3)-N(1,5,5 n+3)+N(0,5,5 n+3)-N(2,5,5 n+3)\} x^{n} \\
& =\sum_{n=0}^{\infty}\{N(0,5,5 n+3)-N(1,5,5 n+3)\} x^{n}+\sum_{n=0}^{\infty}\{N(0,5,5 n+3)-N(2,5,5 n+3)\} x^{n} \\
& =r_{0,1}(3)+r_{0,2}(3) . \\
& \therefore D(x)=r_{0,1}(3)+r_{0,2}(3) . \text { Hence the Corollary. }
\end{aligned}
$$

Corollary 4: Prove that $\frac{\Psi(x)}{x}=r_{2,0}(3)$

Proof: We get,

$$
\begin{aligned}
& \begin{aligned}
& \Psi(x)=-1+\left\{\frac{1}{1-x^{2}}+\frac{x^{5}}{\left(1-x^{2}\right)\left(1-x^{3}\right)\left(1-x^{7}\right)}+\frac{x^{20}}{\left(1-x^{2}\right)\left(1-x^{3}\right)\left(1-x^{7}\right)\left(1-x^{8}\right)\left(1-x^{12}\right)}+\ldots \infty\right\}, \\
&\quad \text { Ramanujan's lost notebook }(1918)]
\end{aligned} \\
& =-1+\left(1+x^{2}+x^{4}+\ldots \infty\right)+x^{5}\left(1+x^{2}+\ldots \infty\right)\left(1+x^{3}+x^{6}+\ldots \infty\right)\left(1+x^{7}+\ldots \infty\right)+\ldots \infty \\
& =x^{2}+x^{4}+x^{6}+x^{7}+2 x^{8}+x^{9}+2 x^{10}+\ldots \infty . \\
& \therefore \frac{\Psi(x)}{x}=x+x^{3}+x^{5}+x^{6}+2 x^{7}+x^{8}+2 x^{9}+\ldots \infty \\
& \quad=\sum_{n=0}^{\infty}\{N(2,5,5 n+3)-N(0,5,5 n+3)\} x^{n} \\
& \quad=r_{2,0}(3) . \\
& \therefore \frac{\Psi(x)}{x}=r_{2,0}(3) . \text { Hence the Corollary. }
\end{aligned}
$$

\section{The generating functions for $\rho_{0}(n)$ and $\rho_{1}(n)$ :}

\subsection{The generating function for $\rho_{0}(n)$ is given below;}

We get $\rho_{0}(n)$ is the number of partitions of $n$ with unique smallest part and all other parts $\leq$ the double of the smallest part given in the Table-14

\begin{tabular}{|l|l|l|}
\hline$n$ & Type of partitions & $\rho_{0}(n)$ \\
\hline 1 & 1 & 1 \\
\hline 2 & 2 & 1 \\
\hline 3 & $3,2+1$ & 2 \\
\hline 4 & 4 & 1 \\
\hline
\end{tabular}




\begin{tabular}{|c|l|c|}
\hline 5 & $5,3+2,2+2+1$ & 3 \\
\hline 6 & $6,4+2$ & 2 \\
\hline 7 & $7,4+3,2+2+2+1$ & 3 \\
$\ldots$ & $\ldots$ & $\ldots$ \\
\hline
\end{tabular}

It is convenient to define $\rho_{0}(0)=0$.

We can write an expression as;

$$
\begin{aligned}
& \rho_{0}(0)+\rho_{0}(1) x+\rho_{0}(2) x^{2}+\rho_{0}(3) x^{3}+\rho_{0}(4) x^{4}+\rho_{0}(5) x^{5}+\rho_{0}(6) x^{6}+\rho_{0}(7) x^{7}+. . \\
& =1 . x+1 \cdot x^{2}+2 \cdot x^{3}+1 \cdot x^{4}+3 \cdot x^{5}+2 \cdot x^{6}+3 \cdot x^{7}+. . \\
& =x+x^{2}+2 x^{3}+x^{4}+3 x^{5}+2 x^{6}+\ldots \infty \\
& \text { or, } \sum_{n=0}^{\infty} \rho_{0}(n) x^{n}=\frac{x}{1-x}+\frac{x^{3}}{\left(1-x^{2}\right)\left(1-x^{3}\right)}+\frac{x^{5}}{\left(1-x^{3}\right)\left(1-x^{4}\right)\left(1-x^{5}\right)}+\ldots \infty \\
& \text { i.e. } \sum_{\mathrm{n}=0}^{\infty} \rho_{0}(n) x^{n}=\sum_{n=0}^{\infty} \frac{x^{2 n+1}}{\left(x^{n+1}\right)_{n+1}} \ldots
\end{aligned}
$$

Now we prove the Theorem, which is known as First Mock Theta Conjecture.

Theorem 1: The number of partitions of $5 \mathrm{n}$ with rank congruent to 1 modulo 5 equals the number of partitions of $5 \mathrm{n}$ with rank congruent to 0 modulo 5 plus the number of partitions of $n$ with unique smallest part and all other parts $\leq$ the double of the smallest part,

i.e. $N(1,5,5 n)=N(0,5,5 n)+\rho_{0}(n)$, where $\rho_{0}(n)$ is the number of partitions of $n$ with unique smallest part and all other parts $\leq$ the double of the smallest part.

Proof: From (4.1a) we get;

$$
\begin{aligned}
& \frac{x}{1-x}+\frac{x^{3}}{\left(1-x^{2}\right)\left(1-x^{3}\right)}+\frac{x^{5}}{\left(1-x^{3}\right)\left(1-x^{4}\right)\left(1-x^{5}\right)}+\ldots \infty=\sum_{n=0}^{\infty} \rho_{0}(n) x^{n} \\
& \Rightarrow x+x^{2}+2 x^{3}+x^{4}+3 x^{5}+2 x^{6}+\ldots \infty=\sum_{n=0}^{\infty} \rho_{0}(n) x^{n} \\
& \Rightarrow 3 \phi(x)+1-A(x)=\sum_{n=0}^{\infty} \rho_{0}(n) x^{n} \text { (By above) } \\
& \Rightarrow 3 r_{1,2}(0)+1-\left(1+r_{0,2}(0)+2 r_{1,2}(0)\right)=\sum_{n=0}^{\infty} \rho_{0}(n) x^{n} \text { (By above) } \\
& \Rightarrow r_{1,2}(0)-r_{0,2}(0)=\sum_{n=0}^{\infty} \rho_{0}(n) x^{n} \text { (By above) } \\
& \Rightarrow \sum_{n=0}^{\infty}\{N(1,5,5 n)-N(2,5,5 n)-N(0,5,5 n)+N(2,5,5 n)\} x^{n}=\sum_{n=0}^{\infty} \rho_{0}(n) x^{n} \\
& \Rightarrow \sum_{n=0}^{\infty}\{N(1,5,5 n)-N(0,5,5 n)\} x^{n}=\sum_{n=0}^{\infty} \rho_{0}(n) x^{n} .
\end{aligned}
$$

Equating the coefficient of $x^{n}$ on both sides, we get; $N(1,5,5 n)=N(0,5,5 n)+\rho_{0}(n)$. Hence the Theorem.

Example 1: For $n=2$, we have;

$N(1,5,10)=9$ with the relevant partitions being: $8+2,6+1+1+1+1,5+3+1+1$, 
$5+2+2+1,4+4+2,4+3+3,3+2+1+1+1+1+1,2+2+2+2+1+1$,

$1+1+1+1+1+1+1+1+1+1$.

But $N(0,5,10)=8$ with the relevant partitions being;

$8+1+1,7+3,5+2+1+1+1,4+4+1+1,4+3+2+1,4+2+2+2$,

$3+1+1+1+1+1+1+1,2+2+2+1+1+1+1$.

Again, $\rho_{0}(2)=1$ with the relevant partition being 2 .

$\therefore N(1,5,10)=N(0,5,10)+\rho_{0}(2)$.

\subsection{The generating function for $\rho_{1}(n)$ is given below;}

We get $\rho_{1}(n)$ is the number of partitions of $n$ with unique smallest part and all other parts $\leq$ one plus the double of the smallest part given in the Table-15

\begin{tabular}{|l|l|l|}
\hline$n$ & Type of partitions & $\rho_{1}(n)$ \\
\hline 1 & 1 & 1 \\
\hline 2 & 2 & 1 \\
\hline 3 & $3,2+1$ & 2 \\
\hline 4 & $4,3+1$ & 2 \\
\hline 5 & $5,3+2,2+2+1$ & 3 \\
\hline$\ldots$ & $\ldots$ & $\ldots$ \\
\hline
\end{tabular}

If we assume $\rho_{1}(0)=0$.

We can write an expression as;

$$
\begin{aligned}
& (0+1)+(1+1) x+(1+1) x^{2}+(2+1) x^{3}+(2+1) x^{4}+(3+1) \cdot x^{5}+. . \\
& =1+2 x+2 x^{2}+3 x^{3}+3 x^{4}+4 x^{5}+4 x^{6}+6 x^{7}+4 x^{8}+\ldots \infty \\
& =\frac{1}{1-x}+\frac{x}{\left(1-x^{2}\right)\left(1-x^{3}\right)}+\frac{x^{2}}{\left(1-x^{3}\right)\left(1-x^{4}\right)\left(1-x^{5}\right)}+\ldots \infty \\
& \text { or, } \sum_{n=0}^{\infty}\left\{\rho_{1}(n)+1\right\} x^{n}=\frac{1}{1-x}+\frac{x}{\left(1-x^{2}\right)\left(1-x^{3}\right)}+\frac{x^{2}}{\left(1-x^{3}\right)\left(1-x^{4}\right)\left(1-x^{5}\right)}+\ldots \infty \\
& \text { i.e. } \sum_{\mathrm{n}=0}^{\infty}\left\{\rho_{1}(n)+1\right\} x^{n}=\sum_{n=1}^{\infty} \frac{x^{n-1}}{\left(x^{n}\right)_{n}} \text {. } \quad \ldots
\end{aligned}
$$

Now we prove the Theorem, which is known as Second Mock Theta Conjecture.

Theorem 2: The double of the number of partitions of $5 n+3$ with rank congruent to 2 modulo 5 equals the sum of the number of partitions of $5 n+3$ with rank congruent to 0 and congruent to 1 modulo 5, and the sum of one and the number of partitions of $n$ with unique smallest part and all other parts $\leq$ one plus the double of the smallest part.

i.e. $2 N(2,5,5 n+3)=N(0,5,5 n+3)+N(1,5,5 n+3)+\rho_{1}(n)+1$, where $\rho_{1}(n)$ is the number of partitions of $n$ with unique smallest part and all other parts $\leq$ one plus the double of the smallest part.

Proof: From (4.2a) we have;

$$
\begin{aligned}
\sum_{n=0}^{\infty}\left\{\rho_{1}(n)+1\right\} x^{n}= & \frac{1}{1-x}+\frac{x}{\left(1-x^{2}\right)\left(1-x^{3}\right)}+\frac{x^{2}}{\left(1-x^{3}\right)\left(1-x^{4}\right)\left(1-x^{5}\right)}+\ldots \infty \\
& =1+2 x+2 x^{2}+3 x^{3}+3 x^{4}+4 x^{5}+4 x^{6}+\ldots \infty \\
& =3 \frac{\Psi(x)}{x}+D(x)
\end{aligned}
$$


$\Rightarrow \sum_{n=0}^{\infty}\left\{\rho_{1}(n)+1\right\} x^{n}=3 \frac{\Psi(x)}{x}+D(x)$, (By above)

$\Rightarrow 3 r_{2,0}(3)+r_{0,1}(3)+r_{0,2}(3)=\sum_{n=0}^{\infty}\left\{\rho_{1}(n)+1\right\} x^{n}$ (By above)

$\Rightarrow \sum_{n=0}^{\infty}\{3 N(2,5,5 n+3)-3 N(0,5,5 n+3)+N(0,5,5 n+3)-N(1,5,5 n+3)+N(0,5,5 n+3)-N(2,5,5 n+3)\} x^{n}$

$$
=\sum_{n=0}^{\infty}\left\{\rho_{1}(n)+1\right\} x^{n}
$$

Equating the coefficient of $x^{n}$ on both sides, we get;

$2 N(2,5,5 n+3)-N(0,5,5 n+3)-N(1,5,5 n+3)=\rho_{1}(n)+1$

$2 N(2,5,5 n+3)=N(0,5,5 n+3)+N(1,5,5 n+3)+\rho_{1}(n)+1$. Hence the Theorem.

Example 2: For $n=1$, we have; $N(2,5,8)=5$ with the relevant partitions being: $8,5+2+1$, $4+4,3+1+1+1+1+1,2+2+2+1+1$.

But $N(1,5,8)=4$ with the relevant partitions being: $5+1+1+1,4+3+1,4+2+2$, $2+2+1+1+1+1$, and $N(0,5,8)=4$ with the relevant partitions are: $7+1,4+2+1+1$, $3+3+2,2+1+1+1+1+1+1$.

Again, $\rho_{1}(1)=1$, with the relevant partition being 1 . Therefore, $2 N(2,5,8)=2 \times 5=10=$ $4+4+1+1 . \quad$ i.e., $2 N(2,5,8)=N(0,5,8)+N(1,5,8)+\rho_{1}(1)+1$.

\section{The Generating Functions For $\beta_{1}(n)$ and $\beta_{2}(n)$ :}

First we shall establish the following identity, which is used in proving the Theorems. If $a$ and $t$ are both real numbers with $|a|<1$ and $|t|<1$, we have;

$$
\begin{aligned}
& \frac{(a t)_{\infty}}{(t)_{\infty}}=\frac{(1-a t)(1-a t x)\left(1-a t x^{2}\right) \ldots \quad \infty}{(1-t)(1-t x)\left(1-t x^{2}\right) \ldots \quad \infty} \\
& =\{(1-a t)(1-a t x) \ldots \infty\}\left(1+t+t^{2}+\ldots \infty\right)\left(1+t x+t^{2} x^{2}+\ldots \infty\right)\left(1+t x^{2}+\ldots \infty\right) \ldots \infty \\
& =1+t\left\{\left(1+x+x^{2}+\ldots \infty\right)-a\left(1+x+x^{2}+\ldots \infty\right)\right\}+t^{2}\left\{\left(1+x+2 x^{2}+2 x^{3}+\ldots \infty\right)-a\left(1+2 x+3 x^{2}+\ldots \infty\right)+\right. \\
& \quad a^{2}\left(x+x^{2}+2 x^{3}+2 x^{4}+\ldots \infty\right)+\ldots \infty \\
& =1+(1-a) t\left(1+x+x^{2}+\ldots \infty\right)+(1-a)(1-a x) t^{2}\left(1+x+2 x^{2}+2 x^{3}+\ldots \infty\right)+\ldots \infty \\
& =1+\frac{(1-a) t}{1-x}+\frac{(1-a)(1-a x) t^{2}}{(1-x)\left(1-x^{2}\right)}+\frac{(1-a)(1-a x)\left(1-a x^{2}\right) t^{3}}{(1-x)\left(1-x^{2}\right)\left(1-x^{3}\right)}+\ldots \infty \\
& =\sum_{n=0}^{\infty} \frac{(a)_{n} t^{n}}{(x)_{n}} \\
& \text { i.e., } \frac{(a t)_{\infty}}{(t)_{\infty}}=\sum_{n=0}^{\infty} \frac{(a)_{n} t^{n}}{(x)_{n}} .
\end{aligned}
$$




\subsection{The generating function for $\beta_{1}(n)$ is defined below;}

We get $\beta_{1}(n)$ is the number of partitions of $n$ into 1 's and parts congruent to 0 or -1 modulo 5 with the largest part $\equiv 0(\bmod 5) \leq 5$ times the number of 1 's given in the Table-16

\begin{tabular}{|l|l|l|}
\hline$n$ & Type of partitions & $\beta_{1}(n)$ \\
\hline 1 & 1 & 1 \\
\hline 2 & $1+1$ & 1 \\
\hline 3 & $1+1+1$ & 1 \\
\hline 4 & $4,1+1+1+1$ & 2 \\
\hline 5 & $1+1+1+1+1$ & 1 \\
\hline 6 & $5+1,1+1+1+1+1+1$ & 2 \\
\hline$\ldots$ & $\ldots$ & $\ldots$ \\
\hline
\end{tabular}

We can write an expression as;

$\beta_{1}(0)+\beta_{1}(1) x+\beta_{1}(2) x^{2}+\beta_{1}(3) x^{3}+\beta_{1}(4) x^{4}+\beta_{1}(5) x^{5}+\beta_{1}(6) x^{6}+.$.

$=1+x+x^{2}+x^{3}+2 x^{4}+x^{5}+2 x^{6}+\ldots \infty$

or, $\sum_{\mathrm{n}=0}^{\infty} \beta_{1}(n) x^{n}=\frac{1}{\left(1-x^{4}\right)\left(1-x^{9}\right)\left(1-x^{14}\right) \ldots}+\frac{x}{\left(1-x^{5}\right)\left(1-x^{9}\right)\left(1-x^{14}\right) \ldots}+\frac{x^{2}}{\left(1-x^{5}\right)\left(1-x^{10}\right)\left(1-x^{14}\right) \ldots}+\ldots \infty$

i.e., $\sum_{n=0}^{\infty} \frac{x^{n}}{\left(x^{5} ; x^{5}\right)_{n}\left(x^{5 n+4} ; x^{5}\right)_{\infty}}=\sum_{n=0}^{\infty} \beta_{1}(n) x^{n}$, it is convenient to define $\beta_{1}(0)=1$.

\subsection{The generating function for $\beta_{2}(n)$ is given below;}

We get $\beta_{2}(n)$ is the number of partitions of $n$ into 2's and all other parts congruent to 0 or -2 modulo 5 with the largest part $\equiv 0(\bmod 5) \leq 5$ times the number of 2 's given in the Table-17

\begin{tabular}{|l|l|l|}
\hline$n$ & Type of partitions & $\beta_{2}(n)$ \\
\hline 1 & none & 0 \\
\hline 2 & 2 & 1 \\
\hline 3 & 3 & 1 \\
\hline 4 & $2+2$ & 1 \\
\hline 5 & none & 0 \\
\hline 6 & $3+3,2+2+2$ & 2 \\
\hline$\ldots$ & $\ldots$ & $\ldots$ \\
\hline
\end{tabular}

We can write an expression as;

$\beta_{2}(0)+\beta_{2}(1) x+\beta_{2}(2) x^{2}+\beta_{2}(3) x^{3}+\beta_{2}(4) x^{4}+\beta_{2}(5) x^{5}+\beta_{2}(6) x^{6}+.$.

$=1+0+x^{2}+x^{3}+x^{4}+2 x^{6}+\ldots \infty$

or, $\sum_{\mathrm{n}=0}^{\infty} \beta_{2}(n) x^{n}=\frac{1}{\left(1-x^{3}\right)\left(1-x^{8}\right)\left(1-x^{13}\right) \ldots}+\frac{x^{2}}{\left(1-x^{5}\right)\left(1-x^{8}\right)\left(1-x^{13}\right) \ldots}+\frac{x^{4}}{\left(1-x^{5}\right)\left(1-x^{10}\right)\left(1-x^{13}\right) \ldots}+\ldots \infty$

i.e., $\sum_{n=0}^{\infty} \frac{x^{2 n}}{\left(x^{5} ; x^{5}\right)_{n}\left(x^{5 n+3} ; x^{5}\right)_{\infty}}=\sum_{n=0}^{\infty} \beta_{2}(n) x^{n}$, it is convenient to define $\beta_{2}(0)=1$. 
Corollary 5: $B(x)=r_{0,2}(1)$

Proof: We get, $B(x)=\prod_{n=1}^{\infty} \frac{\left(1-x^{5 n}\right)}{\left(1-x^{5 n-4}\right)\left(1-x^{5 n-1}\right)} \quad$ [Ramanujan's lost notebook (1916)]

$$
\begin{aligned}
& =\prod_{n=1}^{\infty}\left(1-x^{5 n}\right)\left(1+x^{5 n-4}+\ldots \infty\right)\left(1+x^{5 n-1}+\ldots \infty\right) \\
& =(1-0)+(3-2) x+(12-11) x^{2}+x^{3}+2 x^{4}+\ldots \infty \\
& =\sum_{n=0}^{\infty}\{N(0,5,5 n+1)-N(2,5,5 n+1)\} x^{n}=r_{0,2}(1) .
\end{aligned}
$$

$\therefore B(x)=r_{0,2}(1)$. Hence the Corollary.

Corollary 6: $C(x)=r_{1,2}(2)$

Proof: We get, $C(x)=\prod_{n=1}^{\infty} \frac{\left(1-x^{5 n}\right)}{\left(1-x^{5 n-3}\right)\left(1-x^{5 n-2}\right)}$ [Ramanujan's lost notebook (1916)]

$$
\begin{aligned}
& \qquad=\prod_{n=1}^{\infty}\left(1-x^{5 n}\right)\left(1+x^{5 n-3}+x^{10 n-6}+\ldots \infty\right)\left(1+x^{5 n-2}+x^{10 n-4}+\ldots \infty\right) \\
& =(1-0)+(3-3) x+(16-15) x^{2}+\ldots \infty \\
& =\sum_{n=0}^{\infty}\{N(1,5,5 n+2)-N(2,5,5 n+2)\} x^{n} \\
& =r_{1,2}(2) . \\
& \therefore C(x)=r_{1,2} \text { (2). Hence the Corollary. }
\end{aligned}
$$

Here we give two Theorems, [Andrews et al. (1989)] which are related to the terms $\beta_{1}(n)$ and $\beta_{2}(n)$ respectively.

Theorem 3: The number of partitions of $5 n+1$ with rank congruent to 0 modulo 5 equals the number of partitions of $5 n+1$ with rank congruent to 2 modulo 5 plus the number of partitions of $\mathrm{n}$ into 1 's and all other parts congruent to 0 or -1 modulo 5 with the largest part $\equiv 0(\bmod 5) \leq 5$ times the number of 1 's i.e., $N(0,5,5 n+1)=N(2,5,5 n+1)+\beta_{1}(n)$,

where $\beta_{1}(n)$ is the number of partitions of $n$ into 1 's and all other parts congruent to 0 or -1 modulo 5 with the largest part $\equiv 0(\bmod 5) \leq 5$ times the number of 1 's .

Proof: From (5a) by replacing $\left(z^{-1} x\right)$ for $a$ and $z$ for $t$ we have;

$$
\begin{aligned}
& \frac{(x)_{\infty}}{(z)_{\infty}\left(z^{-1} x\right)_{\infty}}=\frac{1}{\left(z^{-1} x\right)_{\infty}} \sum_{n=0}^{\infty} \frac{\left(z^{-1} x\right)_{n} z^{n}}{(x)_{n}}, \text { where }|z|<1 \text { but } z \neq 0 \\
& =\frac{1}{\left(1-z^{-1} x\right)\left(1-z^{-1} x^{2}\right) \ldots \infty}\left[1+\frac{\left(1-z^{-1} x\right)}{(1-x)}+\frac{\left(1-z^{-1} x\right)\left(1-z^{-1} x^{2}\right) z^{2}}{(1-x)\left(1-x^{2}\right)}+\ldots \infty\right] \\
& \text { or }, \frac{(1-x)\left(1-x^{2}\right) \ldots \infty}{(1-z)(1-z x) \ldots \infty\left(1-z^{-1} x\right)\left(1-z^{-1} x^{2}\right) \ldots \infty}
\end{aligned}
$$




$$
=\frac{1}{\left(1-z^{-1} x\right)\left(1-z^{-1} x^{2}\right) \ldots \infty}+\frac{z}{(1-x)\left(1-z^{-1} x^{2}\right) \ldots \infty}+\frac{z^{2}}{(1-x)\left(1-x^{2}\right)\left(1-z^{-1} x^{3}\right) \ldots \infty}+\ldots \infty
$$

Replacing $x$ by $x^{5}$ and $z$ by $x$, we obtain;

$$
\begin{aligned}
& \frac{\left(1-x^{5}\right)\left(1-x^{10}\right) \ldots \infty}{\left\{(1-x)\left(1-x^{6}\right) \ldots \infty\right\}\left(1-x^{4}\right)\left(1-x^{9}\right) \ldots \infty} \\
& =\frac{1}{\left(1-x^{4}\right)\left(1-x^{9}\right) \ldots \infty}+\frac{x}{\left(1-x^{5}\right)\left(1-x^{9}\right)\left(1-x^{14}\right) \ldots \infty}+\frac{x^{2}}{\left(1-x^{5}\right)\left(1-x^{10}\right)\left(1-x^{14}\right) \ldots \infty}+\ldots \infty \\
& \text { or, } \frac{\left(x^{5} ; x^{5}\right)_{\infty}}{\left(x ; x^{5}\right)_{\infty}\left(x^{4} ; x^{5}\right)_{\infty}}=\sum_{n=0}^{\infty} \frac{x^{n}}{\left(x^{5} ; x^{5}\right)_{n}\left(x^{5 n+4} ; x^{5}\right)_{\infty}} \\
& \text { Hence, } \sum_{n=0}^{\infty} \frac{\left(x^{5} ; x^{5}\right)_{\infty}}{\left(x^{5} ; x^{5}\right)_{n}\left(x^{5 n+4} ; x^{5}\right)_{\infty}}=\frac{\left(1-x^{5 n}\right)}{\left(x ; x^{5}\right)_{\infty}\left(x^{4} ; x^{5}\right)_{\infty}}=\prod_{n=1}^{\infty} \frac{\left(1-x^{5 n-4}\right)\left(1-x^{5 n-1}\right)}{\left(\therefore \sum_{n=0}^{\infty} \beta_{1}(n) x^{n}=B(x)=r_{0,2}(1)\right.} \quad(\text { By above }) \\
& \therefore \sum_{n=0}^{\infty} \beta_{1}(n) x^{n}=r_{0,2}(1)=\sum_{n=0}^{\infty}\{N(0,5,5 n+1)-N(2,5,5 n+1)\} x^{n} .
\end{aligned}
$$

Equating the coefficient of $x^{n}$ on both sides, we get;

$\beta_{1}(n)=N(0,5,5 n+1)-N(2,5,5 n+1)$

i.e., $N(0,5,5 n+1)=N(2,5,5 n+1)+\beta_{1}(n)$. Hence the Theorem.

Example 3: For $n=2$, we have;

$N(0,5,11)=12$ with the relevant partitions being $11,8+2+1,7+4,6+1+1+1+1+1,5+3+1+1+1$, $5+2+2+1+1,4+3+2+2, \ldots$, there are 12 partitions.

And $N(2,5,11)=11$ with the relevant partitions being $9+2,7+1+1+1+1,6+3+1+1,6+2+2+1$, $5+5+1, \ldots$, there are 11 partitions.

Again, $\beta_{1}(2)=1$, with the relevant partition being $1+1$.

$$
\text { i.e., } N(0,5,11)=N(2,5,11)+\beta_{1}(2) \text {. }
$$

Theorem 4: The number of partitions of $5 n+2$ with rank congruent to 1 modulo 5 equals the number of partitions of $5 n+2$ with rank congruent to 2 modulo 5 plus the number of partitions of $\mathrm{n}$ into 2 's and all other parts congruent to 0 or -2 modulo 5 with the largest part $\equiv 0(\bmod 5) \leq 5$ times the number of 1 's. i.e., $N(1,5,5 n+2)=N(2,5,5 n+2)+\beta_{2}(n)$, where $\beta_{2}(n)$ is the number of partitions of $n$ into 2 's and all other parts congruent to 0 or -2 modulo 5 with the largest part $\equiv 0(\bmod 5) \leq 5$ times the number of 2 's.

Proof: From (5a) by replacing $\left(z^{-1} x\right)$ for $a$, and $z$ for $t$ we have;

$\frac{(x)_{\infty}}{(z)_{\infty}\left(z^{-1} x\right)_{\infty}}=\frac{1}{\left(z^{-1} x\right)_{\infty}} \sum_{n=0}^{\infty} \frac{\left(z^{-1} x\right)_{n} z^{n}}{(x)_{n}}$, where $|z|<1$ but $z \neq 0$ 


$$
\begin{gathered}
=\frac{1}{\left(1-z^{-1} x\right)\left(1-z^{-1} x^{2}\right) \ldots \infty}\left[1+\frac{\left(1-z^{-1} x\right) z}{(1-x)}+\frac{\left(1-z^{-1} x\right)\left(1-z^{-1} x^{2}\right) z^{2}}{(1-x)\left(1-x^{2}\right)}+\ldots \infty\right] \\
o r, \frac{(1-x)\left(1-x^{2}\right) \ldots \infty}{(1-z)(1-z x) \ldots \infty\left(1-z^{-1} x\right)\left(1-z^{-1} x^{2}\right) \ldots \infty} \\
=\frac{1}{\left(1-z^{-1} x\right)\left(1-z^{-1} x^{2}\right) \ldots \infty}+\frac{z}{(1-x)\left(1-z^{-1} x^{2}\right) \ldots \infty}+\frac{z^{2}}{(1-x)\left(1-x^{2}\right)\left(1-z^{-1} x^{3}\right) \ldots \infty}+\ldots \infty
\end{gathered}
$$

We get by replacing $x$ by $x^{5}$, and $z$ by $x^{2}$;

$\frac{\left(1-x^{5}\right)\left(1-x^{10}\right)\left(1-x^{15}\right) \ldots \infty}{\left\{\left(1-x^{2}\right)\left(1-x^{7}\right) \ldots \infty\right\}\left\{\left(1-x^{3}\right)\left(1-x^{8}\right) \ldots \infty\right\}}$

$=\frac{1}{\left(1-x^{3}\right)\left(1-x^{8}\right) \ldots \infty}+\frac{x^{2}}{\left(1-x^{5}\right)\left(1-x^{8}\right)\left(1-x^{13}\right) \ldots \infty}+\frac{x^{4}}{\left(1-x^{5}\right)\left(1-x^{10}\right)\left(1-x^{13}\right) \ldots \infty}+\ldots \infty$

or, $\frac{\left(x^{5} ; x^{5}\right)_{\infty}}{\left(x^{2} ; x^{5}\right)_{\infty}\left(x^{3} ; x^{5}\right)_{\infty}}=\sum_{n=0}^{\infty} \frac{x^{2 n}}{\left(x^{5} ; x^{5}\right)_{n}\left(x^{5 n+3} ; x^{5}\right)_{\infty}}$.

Hence, $\sum_{n=0}^{\infty} \frac{x^{2 n}}{\left(x^{5} ; x^{5}\right)_{n}\left(x^{5 n+3} ; x^{5}\right)_{\infty}}=\frac{\left(x^{5} ; x^{5}\right)_{\infty}}{\left(x^{2} ; x^{5}\right)_{\infty}\left(x^{3} ; x^{5}\right)_{\infty}}=\sum_{n=1}^{\infty} \frac{\left(1-x^{5 n}\right)}{\left(1-x^{5 n-2}\right)\left(1-x^{5 n-3}\right)}$

$$
\begin{aligned}
\therefore \sum_{n=0}^{\infty} \beta_{2}(n) x^{n} & =C(x) \quad \text { (By above) } \\
\therefore \sum_{n=0}^{\infty} \beta_{2}(n) x^{n} & =r_{1,2}(2),(\text { By above) } \\
& =\sum_{n=0}^{\infty}\{N(1,5,5 n+2)-N(2,5,5 n+2)\} x^{n} .
\end{aligned}
$$

Equating the coefficient of $x^{n}$ on both sides, we get;

$$
\begin{aligned}
& \beta_{2}(n)=N(1,5,5 n+2)-N(2,5,5 n+2) . \\
& \quad \therefore N(1,5,5 n+2)=N(2,5,5 n+2)+\beta_{2}(n) . \quad \text { Hence the Theorem. }
\end{aligned}
$$

Example 4: For $n=2$, we have;

$N(1,5,12)=16$ with the relevant partitions being $9+2+18+4,7+1+1+1+1+1,6+3+1+1+1$, $5+4+2+1,4+4+4, \ldots$, , there are 16 partitions.

And $N(2,5,12)=15$ with the relevant partitions being $10+1+1,9+3,7+2+1+1+1,6+3+2+1$, $6+2+2+2,6+4+1+1, \ldots$, there are 15 partitions.

Again, $\beta_{2}(2)=1$, with the relevant partition being 2 .

$N(1,5,12)=16, N(2,5,12)=15, \beta_{2}(2)=1$, with the relevant partition being 2 .

$$
\therefore N(1,5,12)=N(2,5,12)+\beta_{2}(2) \text {. }
$$

\section{CONCLUSIONS}

We have proved for any positive integer of $n$ in two Theorems first and second mock theta conjectures. But we have verified these for $n=2$ and 1 respectively.

We have also verified last two Theorems in terms of $\beta_{1}(n)$ and $\beta_{2}(n)$ respectively for $n=2$. 


\section{REFERENCES}

[1] G. E. Andrews and F.G. Garvan, Ramanujan's Lost Notebook VI: Mock Theta Conjectures, Advance in Mathematics, 73,(1989),pp.242-255.

[2] G. E. Andrews, An Introduction to Ramanujan's Lost Notebook, American Mathematical Monthly, 86, (1979),pp. 89-108.

[3] A.O.L. Atkin and P.Swinnerton-Dyer, Some Properties of Partitions, Proc. London Math., Soc. (3), 4, (1954), pp. 84-106.

[4] F. Dyson, Some guesses in the theory of partitions, Eureka,Cambridge,U.K., 8, (1944),pp.10-15.

[5] F.G. Garvan, Dyson's Rank Function and Andrews's SPT- Function, fgarvan@ufl.edu, Math.Talks, Tianjin, (2013),pp. 1-43.

[6] F.G. Garvan, Combinatorial interpretation of Ramanujan partition congruences, Trans, Amer. Math. Soc. 305, (1988), pp.47-77.

[7] S. Ramanujan, The lost notebook and other unpublished papers, Spriger-Verlag, (1988), pp.1-76.

[8] S. Ramanujan, The lost notebook and other unpublished papers, (Intro. By G. E. Andrews), Norosa, New Delhi, (1988), pp. 01-76.

[9] S. Ramanujan, The lost notebook and other unpublished papers, (1918), pp.1-200. 